\title{
Ictal SPECT in Diagnosis of Non-Ketotic Hyperglycemia-Related Seizure Manifesting as Speech Arrest
}

\author{
Kyung Wook Kang ${ }^{\text {a* }}$ \\ Sang-Hoon Kim ${ }^{\text {a* }}$ \\ Jae-Myung $\mathrm{Kim}^{\mathrm{a}}$ \\ Tai-Seung Nam ${ }^{a}$ \\ Kang-Ho Choi \\ Myeong-Kyu Kim ${ }^{\mathrm{a}, \mathrm{c}}$ \\ aDepartment of Neurology, \\ Chonnam National University Hospital, \\ Gwangju, Korea \\ bDepartment of Neurology, \\ Chonnam National University \\ Medical School, \\ Chonnam National University \\ Hwasun Hospital, Hwasun, Korea \\ 'Department of Neurology, \\ Chonnam National University \\ Medical School, Gwangju, Korea
}

Received July 24, 2018

Revised October 15, 2018

Accepted October 17, 2018

\section{Correspondence}

Myeong-Kyu Kim, MD, PhD

Department of Neurology, Chonnam National University

Medical School, 42 Jebong-ro, Dong-gu, Gwangiu 61469, Korea

Tel +82-62-220-6161

Fax +82-62-228-3461

E-mail mkkim@chonnam.ac.kr

*These authors contributed equally to this work.
Dear Editor,

Out of various neurological manifestations associated with non-ketotic hyperglycemia $(\mathrm{NKH}),{ }^{1}$ speech arrest as a manifestation of seizure is very rare although visual and generalized seizures have been often reported. ${ }^{2,3}$ We report herein a patient with speech arrest as a manifestation of NKH-induced seizure in which technetium-99m (Tc-99m) hexamethylpropylene amine oxime (HMPAO) brain single-photon emission computed tomography (SPECT) revealed a localized seizure focus.

A right-handed, 74-year-old man who had a past medical history of type 2 diabetes mellitus (DM) and no epileptic seizure presented with several episodes of speech arrest and right lower facial spasm without motor weakness of extremities for 5 days before visit. The average duration in each episode was $2 \mathrm{~min}$, during which his consciousness and auditory comprehension were considered in the normal range of characteristics. However, he could not speak out loud at all. He did not have no history of fever or recent flu-like symptoms or head injury. The patient had been prescribed valproate for 4 days after being diagnosed with focal aware seizure at a local clinic without relevant electroencephalography (EEG) findings at that time.

The laboratory data showed elevated serum glucose level of $446 \mathrm{mg} / \mathrm{dL}$ and hemoglobin A1c of $11.4 \%$. The EEG showed mainly muscle artifacts due to a facial spasm, which obscured the presence of epileptiform discharges, but intermittent left frontotemporal fast rhythmic activities during ictal phase (Fig. 1A). The magnetic resonance imaging and angiography of the brain was normal. The Tc-99m HMPAO brain SPECT demonstrated hyper-perfusion at the left precentral gyrus and, middle and inferior frontal gyrus (Fig. 1B). Our patient was diagnosed with $\mathrm{NKH}$-induced seizure and aggressive glucose control and oral carbamazepine was done. The patient had become seizure free since the second day after hospitalization.

We report a rare case of NKH-induced focal aware seizure presenting with right facial twitching and speech arrest. Although the pathogenesis of epileptic seizure in patients with $\mathrm{NKH}$ is not fully understood, it is probable that elevated serum glucose level is a proconvulsant condition. ${ }^{4}$ Hyperglyceima also increases gamma-aminobutyric acid metabolism, and thereby lowers the seizure threshold. ${ }^{1}$ Therefore, the early diagnosis and immediate blood sugar control seems to be important in case of seizure associated with NKH. However, the clinical manifestations may be obscure as in our case or other previously reported cases ${ }^{1,5}$ which poses a challenge to diagnosis.

Penfield and Rasmussen ${ }^{6}$ previously showed that electrical stimulation of the dominant frontal operculum, and both precentral gyri caused speech arrest. In addition, one recent functional mapping study using direct cortical stimulation showed that speech arrest is most likely to occur with stimulation of the posterior inferior frontal gyrus and precentral gyrus. ${ }^{7}$

@ This is an Open Access article distributed under the terms of the Creative Commons Attribution Non-Commercial License (https://creativecommons.org/licenses/by-nc/4.0) which permits unrestricted non-commercial use, distribution, and reproduction in any medium, provided the original work is properly cited. 

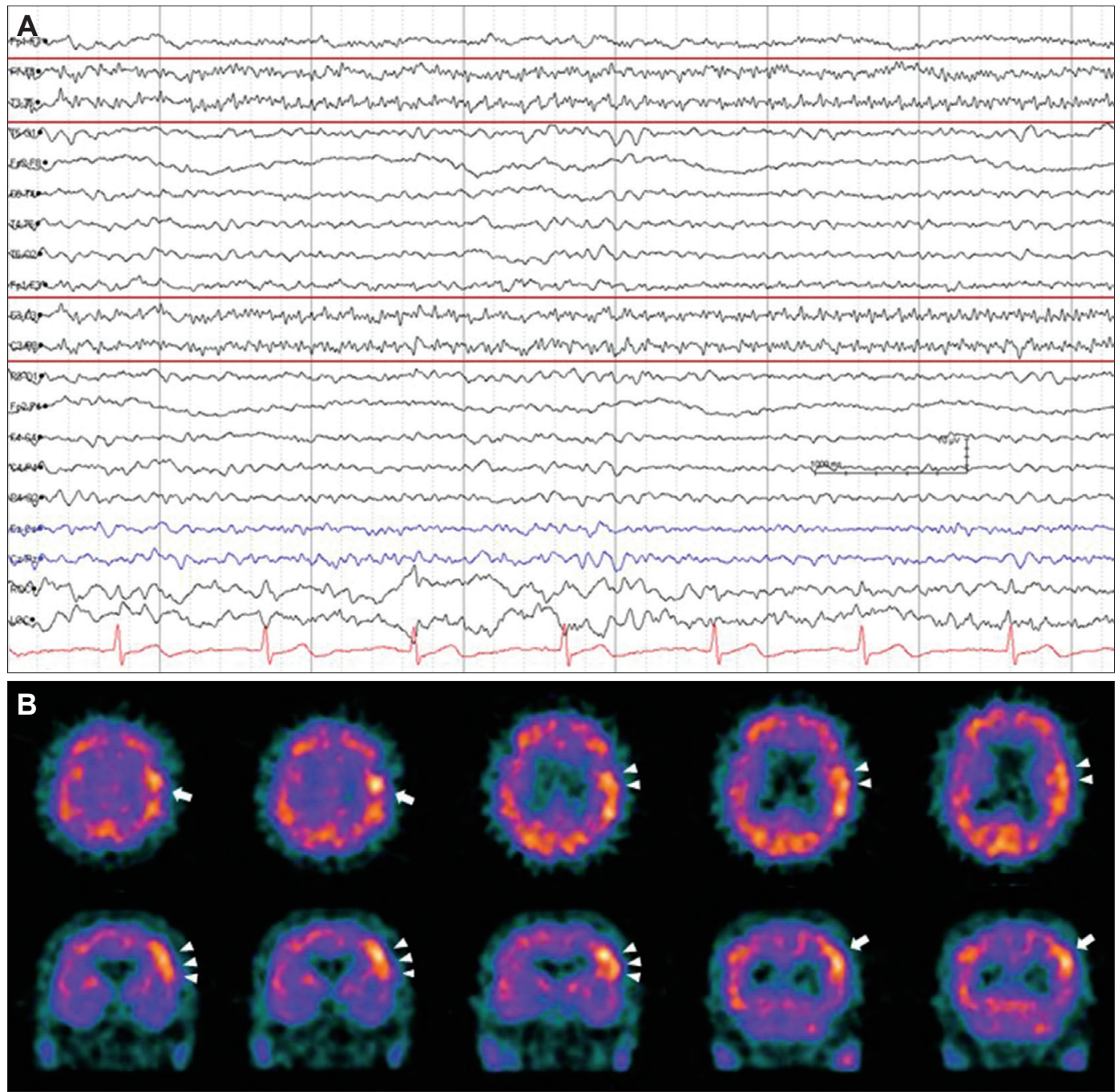

Fig. 1. Ictal EEG and brain SPECT. A: Ictal EEG shows focal rhythmic epileptic discharges over the left frontotemporal area. B: Ictal brain SPECT shows focal hyperperfusion at left precentral gyrus (arrows) and middle and inferior frontal gyri (arrowheads). EEG: electroencephalography, SPECT: singlephoton emission computed tomography.

In other words, the above mentioned areas are considered as negative motor area (NMA) of speech.

In our patient, the ictal SPECT was revealed increased perfusion in the areas consistent with the previous reports. Speech arrest was observed simultaneously with right lower facial twitching due to excitation of the motor cortex in accordance with the motor homunculus. Similarly, previous cases reported sites producing both inhibition of ongoing movements and also excitation of facial musculature. ${ }^{8}$

Although some cases of NKH-associated occipital lobe seizures diagnosed through brain SPECT had been previously reported, ${ }^{5}$ to the best our knowledge, we report the first case of $\mathrm{NKH}$-induced focal aware seizure presenting with speech arrest in which the relevant anatomical regions were demonstrated by the brain SPECT.

Our report demonstrated that ictal SPECT should be con- sidered as one of diagnostic tool in patients with a suspected $\mathrm{NKH}$-induced speech arrest as an early recognition of seizure and rapid correction of blood sugar are critical.

Conflicts of Interest

The authors have no potential conflicts of interest to disclose.

\section{Acknowledgements}

This work was supported by Basic Science Research Program through the National Research Foundation of Korea (NRF) funded by the Ministry of Education (NRF-2018R1D1A1B07048873).

\section{REFERENCES}

1. Hennis A, Corbin D, Fraser H. Focal seizures and non-ketotic hyperglycaemia. J Neurol Neurosurg Psychiatry 1992;55:195-197.

2. Carril JM, Guijarro C, Portocarrero JS, Solache I, Jiménez A, Valera de Seijas E. Speech arrest as manifestation of seizures in non-ketotic hyperglycaemia. Lancet 1992;340:1227. 
Neurol Psychiatry 1949;61:21-27.

3. Venna N, Sabin TD. Tonic fecal seizures in nonketotic hyperglycemia of diabetes mellitus. Arch Neurol 1981;38:512-514.

4. Schwechter EM, Velísková J, Velísek L. Correlation between extracellular glucose and seizure susceptibility in adult rats. Ann Neurol 2003; 53:91-101.

5. Liu CJ, Tsai HH, Ko KY, Lu CC, Yen RF. Ictal phase perfusion SPECT of nonketotic hyperglycemia-induced parieto-occipital seizure. Clin Nucl Med 2017;42:e67-e68.

6. Penfield W, Rasmussen T. Vocalization and arrest of speech. Arch tex 2012;48:1251-1261.
7. Chang EF, Breshears JD, Raygor KP, Lau D, Molinaro AM, Berger MS. Stereotactic probability and variability of speech arrest and anomia sites during stimulation mapping of the language dominant hemisphere. J Neurosurg 2017;126:114-121.

8. Filevich E, Kühn S, Haggard P. Negative motor phenomena in cortical stimulation: implications for inhibitory control of human action. Cor- 